\title{
Phase Transition Behavior of HPMC-AA and Preparation of HPMC-PAA Nanogels
}

\author{
Risheng Yao, Jiajia Xu, Xihua Lu, and Shengsong Deng \\ Pharmaceutical Engineering School, Hefei University of Technology, Hefei 230009, China \\ Correspondence should be addressed to Risheng Yao,yaors@163.com
}

Received 3 August 2010; Revised 21 October 2010; Accepted 1 November 2010

Academic Editor: Xiaogong Wang

Copyright ( $\odot 2011$ Risheng Yao et al. This is an open access article distributed under the Creative Commons Attribution License, which permits unrestricted use, distribution, and reproduction in any medium, provided the original work is properly cited.

The lower critical solution temperature (LCST) of hydroxypropyl methylcellulose (HPMC) under mixing with acrylic acid (AA) monomer has been studied by turbidity measurements. It has been found that the LCST of the HPMC was drastically reduced from $60^{\circ} \mathrm{C}$ to $38^{\circ} \mathrm{C}$ with the increase of the concentration of AA, while the HPMC is kept at $0.5 \mathrm{wt} \%$. The driving force shifting the LCST is attributed to the hydrogen bonding and hydrophobic interaction of the molecules. Then surfactant-free HPMC-PAA nanogels have been synthesized via the polymerization of AA monomer with the collapsed HPMC as a template or core at their LCST, using KPS and TEMED as redox initiator in the presence of BIS as cross-linking agent. HPMC-PAA nanogels have 50 150 $\mathrm{nm}$ diameters characterized by transmission electron microscope and dynamic light scattering. The HPMC-PAA nanogels exhibit the temperature phase transition behaviors, and these nanogels' volume phase transition temperature is close to the LCST of HPMC/AA system.

\section{Introduction}

PAA nanogels and PAA microparticles are used for many drug release controlling materials for the entrapment of hydrophilic drug candidates. Hydrophilic nanogels are usually synthesized via inverse microemulsion polymerization. However, it is very difficult to completely remove organic solvent and surfactant from resulting nanogels; consequently, these nanoparticles are all not suitable for drug delivery systems [1]. Then there is a growing interest in the formation of polymer complexes between a proton-accepting polymer such as poly (ethylene oxide) (PEO) and the protondonating polymer poly (acrylic acid) (PAA) via $\mathrm{H}$-bonding in aqueous media as a model of biological systems [26]. But, only a few studies have been reported so far on the effect of complexation on the lower critical solution temperature (LCST) of a nonionic polymer $[7,8]$. Budtova and coworkers investigated the formation and properties of the interpolymer complexation between PAA and cellulose ethers including hydroxyethylcellulose (HEC) and methylcellulose (MC) [9-11]. Lu and coworkers had done a lot of research about the phase transition behavior of the HPC/PAA complex with change of concentration and $\mathrm{pH}$ [12-16]. Their experimental results showed that the mixtures of the cellulose ethers and nonionized PAA in aqueous solution formed interpolymer complexes due to hydrogen bonding which is the root cause of the reduced LCST. In fact, the hydroxypropyl methylcellulose (HPMC) also exhibits a lower critical solution temperature (LCST) at $60^{\circ} \mathrm{C}$, and a remarkable hydration-dehydration would occur in aqueous solution in response to relatively small changes in temperature around the LCST. Moreover, the HPMC is one of the cellulose ethers approved by the United States Food and Drug Administration (FDA) for the use in food, drug, cosmetics, and building materials [17]. To our knowledge, the studies of the phase transition behavior of HMPC which mixed them with the small monomers as proton-donating molecule have not been reported.

In this work, the lower critical solution temperature (LCST) of the HPMC in presence of acrylic acid (AA) was studied. It was found that the LCST of the HPMC was drastically reduced after adding AA into the HPMC solution, and a new synthesis method was used to synthesize surfactant-free PAA/HMPC nanogels directly in an aqueous 

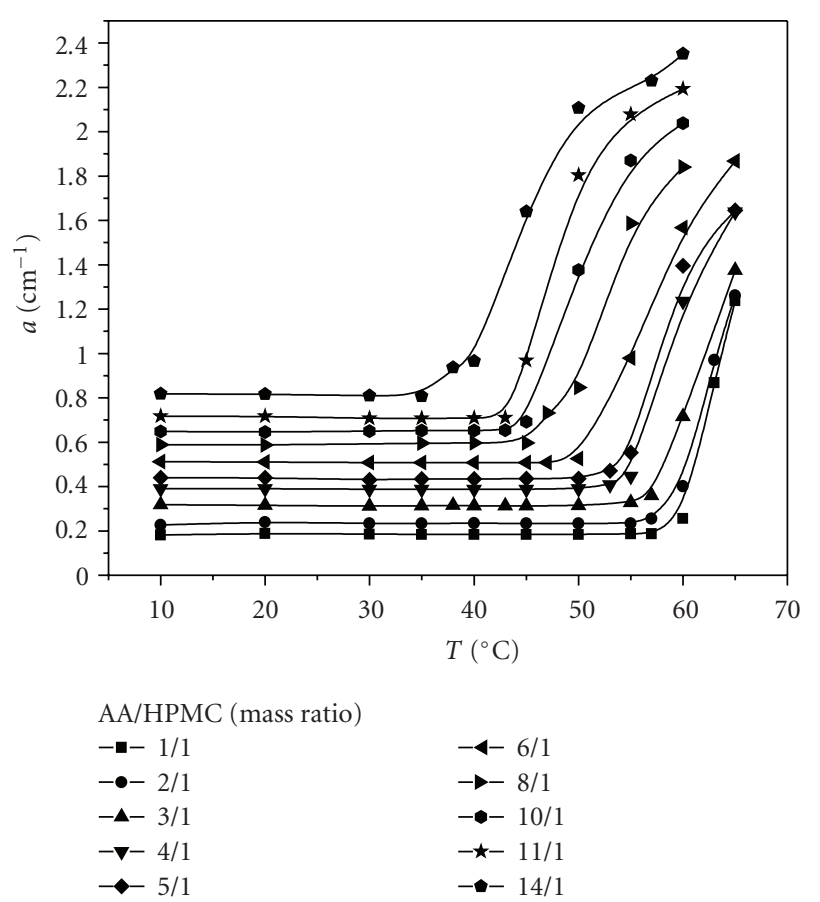

(a)

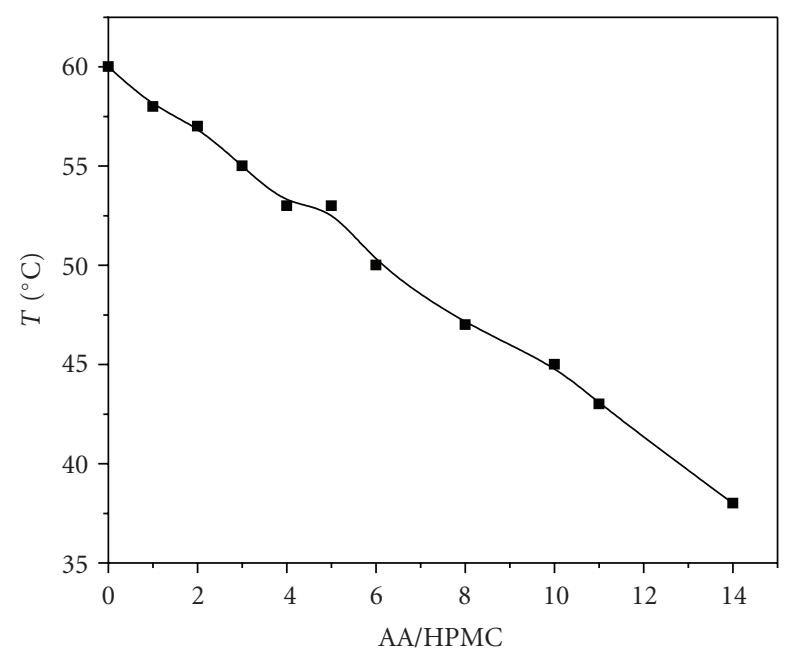

(b)

Figure 1: (a) Temperature-dependent turbidity of the HPMC/AA complex at the different mass ratios of AA to HPMC. (b) The LCST of the different mass ratios of AA to HPMC (the concentration of HPMC is $0.5 \mathrm{wt} \%$ ).

system. The temperature phase transition behavior and diameter of the nanogels were carried out using different analytical techniques such as turbidity, transmission electron microscope (TEM), and dynamic laser light scattering (DLS) measurements.

\section{Experimental}

2.1. Materials. Dry Hydroxypropyl Methylcellulose (HPMC) powders (average $\left.\mathrm{Mw}=1.0 \times 10^{5}\right)$, acrylic acid $(\mathrm{AA})$,

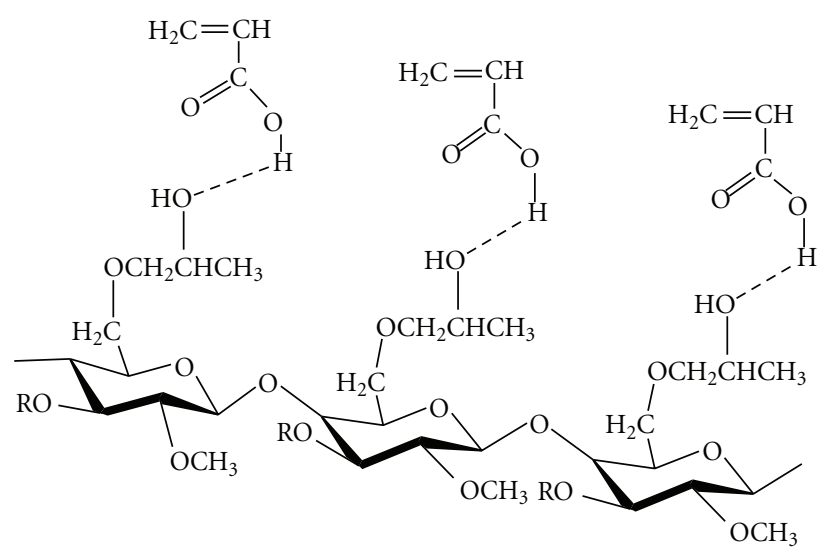

FIGURE 2: Schematic hydrogen bonding between the hydroxypropyl methylcellulose (HPMC) and acrylic acid (AA).

$\mathrm{N}, \mathrm{N}^{\prime}$-methylenebisacrylamide (BIS), N,N, $\mathrm{N}^{\prime}, \mathrm{N}^{\prime}$-tetramethylethylenediamine (TEMED), and potassium peroxodisulfate (KPS) were purchased from Tianjin Bodi Chemical Ltd. Deionized and distilled water was used throughout.

2.2. Sample Preparation. The HPMC powder was dissolved in deionized water at a concentration of $0.5 \mathrm{wt} \%$ at 50 $60^{\circ} \mathrm{C}$. The solution clarified after cooling. The AA solution is analytical grade. These two solutions were then mixed to produce the desired concentrations studied in this paper.

2.3. HPMC-PAA Hydrogel Synthesis. The polymerization and cross-linking reaction for different compositions of AA/HPMC system were carried out in glass vessel at their respective LCSTs for $4-5 \mathrm{~h}$ by using KPS and TEMED as redox initiator $(1.0 \mathrm{wt} \%$ based on $\mathrm{AA})$ in the presence of crosslinking agent $\mathrm{BIS}$ ( $1.0 \mathrm{wt} \%$ based on AA). The whole reaction process was under the protection of the $\mathrm{N}_{2}$.

2.4. Turbidity Measurement. The insoluble material concentration can scatter light and reduce the transmission of visible light. The turbidity $(a)$ is defined as the reduction in fractional light intensity per unit penetration length in the sample. In this experiment, the turbidity was measured using a spectrophotometer (Shanghai Xinmao Ltd. UV-7504) at the maximum absorption wavelength of the incident light of $295 \mathrm{~nm}$. The temperature of HPMC-AA water solution or HPMC-PAA nanogel dispersion was controlled by a circulation water bath (Shanghai Experimental Instruments. model 501). The temperature of the sample was measured using an electric contact thermometer. The sample was allowed to reach equilibrium for $30 \mathrm{~min}$ or longer after adjusting each temperature.

2.5. Transmission Electron Micrograph Characterization. TEM of the HPMC-PAA complex nanogel particles was taken using an H-800 electron microscope (Hitachi, Tokyo, Japan). The TEM sample was prepared by placing a dilute 


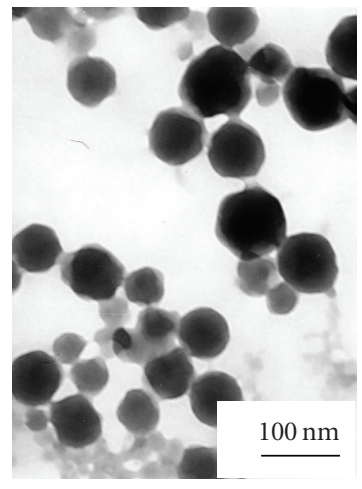

(a)

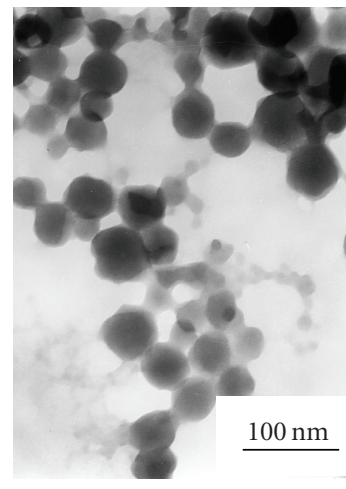

(b)

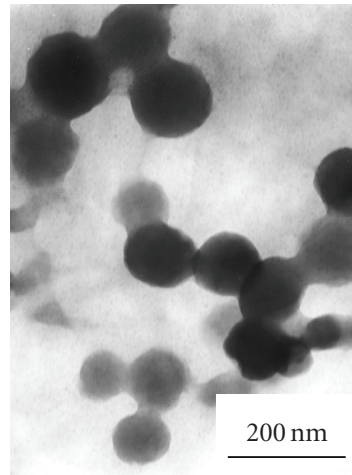

(c)

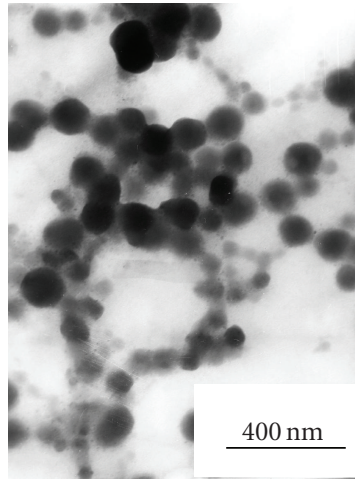

(d)

FIgURE 3: Micrographs of HPMC/PAA nanoparticles in water by transmission electron microscope: the mass ratio of AA to HPMC is (a) 1, (b) 6 , (c) 10 , and (d) 14 (the concentration of HPMC is $0.5 \mathrm{wt} \%$ ).

drop of the measured solution onto the copper grids and allowing it to dry.

2.6. Fourier Transform Infrared Spectroscopy. Fourier transform infrared spectroscopy (FTIR) spectra were measured on a Perkins-Elmer FTIR spectrophotometer. The HPMC-PAA gel particle suspension was lyophilized to dry powder, and the obtained dried powder was mixed with $\mathrm{KBr}$ and pressed to a plate for measurement.

2.7. Dynamic Light Scattering Characterization. The size distribution of the nanoparticles was determined by dynamic light scattering using a Malvern Instruments Zetasizer Nano series instrument (ZS90) equipped with a $22 \mathrm{~mW} \mathrm{He}-\mathrm{Ne}$ laser operating at a wavelength of $633 \mathrm{~nm}$. Measurements were carried out at $25^{\circ} \mathrm{C}$. Dispersion Technology Software 5.03 (Malvern Instruments) was used to record and analyze the data to determine particle size distribution.

\section{Results and Discussion}

3.1. Effect of AA Concentrations on the LCST of HPMC. Figure 1(a) shows the turbidity of the HPMC-AA complex in aqueous solution with different mass ratios of AA to HPMC as a function of temperature. Figure 1(b) summarizes the variation of the LCST as a function of AA concentration. The LCST of $0.5 \mathrm{wt} \%$ HPMC aqueous solution shifted to $38^{\circ} \mathrm{C}$ as AA concentration was up to $7 \mathrm{wt} \%$. The decrease of the phase transition temperature is attributed to the formation of hydrogen bonding between the $-\mathrm{COOH}$ group of the AA and $-\mathrm{OH}$ group of the HPMC. The hydrogen bonds between the $-\mathrm{COOH}$ group of the AA and $-\mathrm{OH}$ group of the HPMC are more stable than those between the $-\mathrm{OH}$ groups of water and HPMC, and the hydrophobicity of the HPMC chains increases with the increase in the volume of AA.

HPMC in water has the LCST at approximately $60^{\circ} \mathrm{C}$, while acrylic acid (AA) is a hydrophilic molecule and readily dissolves in water. The phase transition of the HMPC is related to the delicate hydrophilic-hydrophobic balance of HPMC configuration. When the HPMC and AA monomer are mixed, a hydrogen bonding between the HPMC and AA

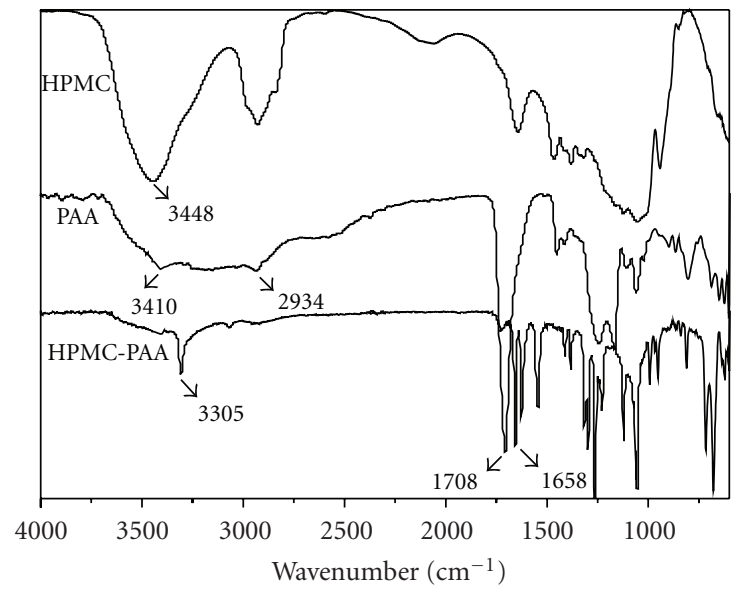

FIGURE 4: Fourier transform infrared spectroscopy of HPMC, PAA, and HPMC-PAA particles.

can form as shown in Figure 2. That is, the AA acts as a proton donor interacting with a proton acceptor (HPMC) to give a complex in which decreasing the LCST of the hydrophilic HPMC resulted in the phase transition at a lower temperature. The steric repulsion between hydrophilic groups of HPMC chain and AA monomer accounts for the stability of the aggregates [12].

3.2. Formation of PAA/HPMC Nanoparticles. There was no particle observed by TEM at room temperature in the aqueous solution of AA and HPMC system. As the AA polymerized in different ratios of AA/HPMC aqueous solution at their respective LCSTs, a large number of nanospheres were observed by TEM at room temperature, as seen in Figure 3. Therefore, the formation of the HPMC/PAA complex nanogels is due to the polymerization of $\mathrm{AA}$ monomer with the collapsed HPMC as a template or core at their LCST. The nanogels were $50 \sim 150 \mathrm{~nm}$ in diameters.

Figure 4 shows the FTIR spectra of HPMC, PAA, and HPMC-PAA gel particles. For pure PAA, the peak centered at $1708 \mathrm{~cm}^{-1}$ was assigned to the associated carboxylic 


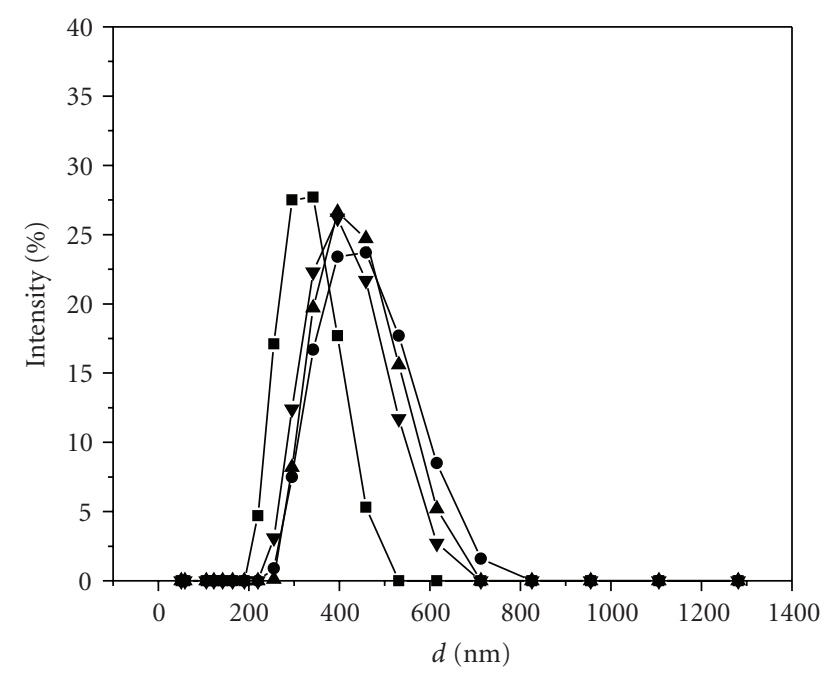

AA/HPMC (mass ratio)

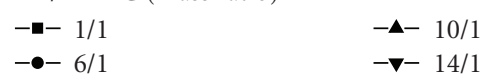

Figure 5: The size distributions of HPMC/PAA nanoparticles in water by dynamic light scattering.
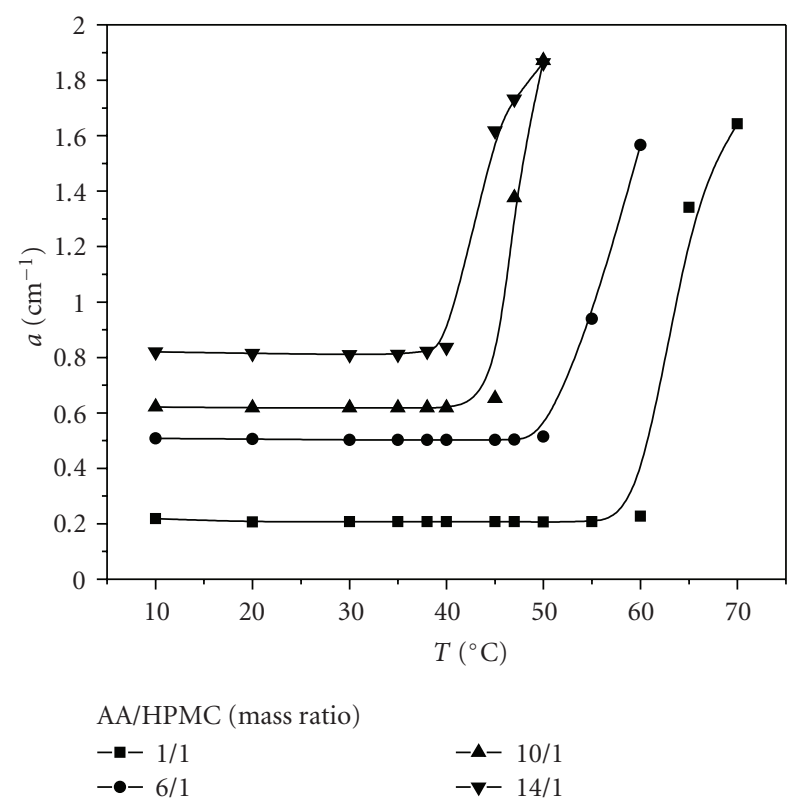

Figure 6: Temperature-dependent turbidity of the HPMC-PAA nanogels at the different mass ratios of AA to HPMC.

acid groups [18], suggesting that most of the carboxylic acid groups were associated with intramolecular hydrogen bonding. In HPMC-PAA particles, the absorption peak of carbonyl groups moves to $1658 \mathrm{~cm}^{-1}$, indicating intermolecular hydrogen bonds formed between PAA and HPMC at the expense of partial detachment of intramolecular hydrogen bonds among the carboxylic groups of PAA. Changes in the hydroxyl stretching region were also observed. For pure HPMC, the absorption band of hydroxyl group at $3448 \mathrm{~cm}^{-1}$
TABLE 1: The nanosphere characterisation results of HPMC/PAA nanoparticles by DLS.

\begin{tabular}{lccc}
\hline Nanogel & $\begin{array}{c}\text { Size } \\
\text { distribution } \\
(\mathrm{nm})\end{array}$ & $\begin{array}{c}\text { Peak } \\
\text { diameter } \\
(\mathrm{nm})\end{array}$ & $\begin{array}{c}\text { Average } \\
\text { diameter } \\
(\mathrm{nm})\end{array}$ \\
\hline $\mathrm{AA} / \mathrm{HPMC}=1$ & $190.1 \sim 531.2$ & 324.3 & 370.3 \\
$\mathrm{AA} / \mathrm{HPMC}=6$ & $220.2 \sim 825$ & 440.6 & 469.4 \\
$\mathrm{AA} / \mathrm{HPMC}=10$ & $220.2 \sim 712.4$ & 424.9 & 499.2 \\
$\mathrm{AA} / \mathrm{HPMC}=14$ & $220.2 \sim 712.4$ & 402.4 & 502.3 \\
\hline
\end{tabular}

shifts to $3305 \mathrm{~cm}^{-1}$ in HPMC-PAA particles. In PAA spectrum, the broad intensive bands at 3410 and $2934 \mathrm{~cm}^{-1}$ can be observed. These two bands are attributable to the free hydroxyl groups and the hydroxyl groups suffering from hydrogen bonds, respectively [19]. For HPMC-PAA nanogel particles, the intensity of the band at $2934 \mathrm{~cm}^{-1}$ decreases significantly, and the band at $3410 \mathrm{~cm}^{-1}$ shifts to a lower wave number $\left(3305 \mathrm{~cm}^{-1}\right)$. The intensity decrease in the band at $2934 \mathrm{~cm}^{-1}$ is caused by the hydrogen bonding between HPMC and PAA instead of self-associated hydrogen bonding in PAA itself, while the shift of the band corresponding to free hydroxyl groups of PAA again indicates that interpolymer hydrogen bonding between PAA and HPMC is formed in the particles. So the obtained HPMC-PAA gel particles have a semi-IPN structure.

The particle sizes were analyzed by dynamic light scattering (DLS). Figure 5 shows the size distributions of the HPMC-PAA nanogels by intensity, and the results are summarized in Table 1 . We see that the average sizes and distributions of these particles measured by DLS are bigger than those observed by TEM, and the nanogels almost have an average diameter ranging from $370 \mathrm{~nm}$ to $500 \mathrm{~nm}$. A bigger particle size by the means of DLS is due to the swelling state of the nanogel in water, while the particle is in dry state by the means of TEM.

3.3. The Temperature Phase Transition Behaviors of HPMCPAA Nanogels. Like its polymer chain counterpart, the resultant surfactant-free HPMC-PAA nanogels exhibit the temperature volume phase transition behaviors. The HPMCPAA nanogels undergo a volume phase transition in water from the swollen state to the collapsed state as the temperature increases. Figure 6 shows the turbidity of HPMCPAA nanogels solution as a function of temperature at the different mass ratios of AA to HPMC. The curves indicate that the nanogels exhibit the volume phase transition above their LCST similar to the uncrosslinked HPMC/AA aqueous solution at the given concentration. The volume phase transition of the HPMC-PAA nanogel also occurs at about $38^{\circ} \mathrm{C}$ when the mass ratio of AA to HPMC is about 14 (see Figures 1 and 6). It may be due to the equally strong hydrogen bonding interaction between the $-\mathrm{COOH}$ of the PAA generated and $-\mathrm{OH}$ of the HPMC. The driving forces for the thermo-sensitive volume phase transition could be considered to be a balance between the hydrophilic and hydrophobic interactions of inter-HPMC and intra-HMPC. 


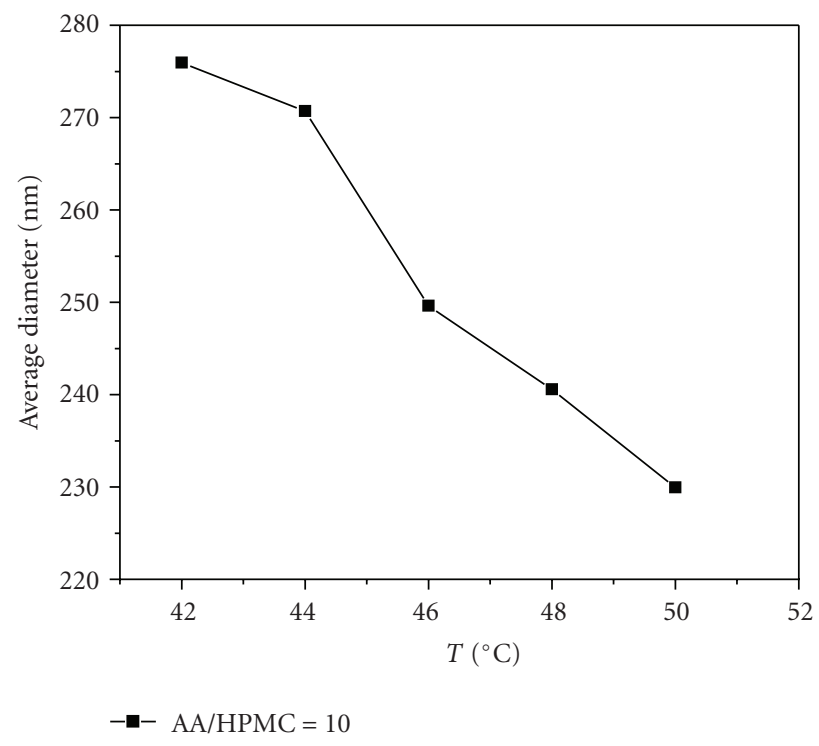

(a)

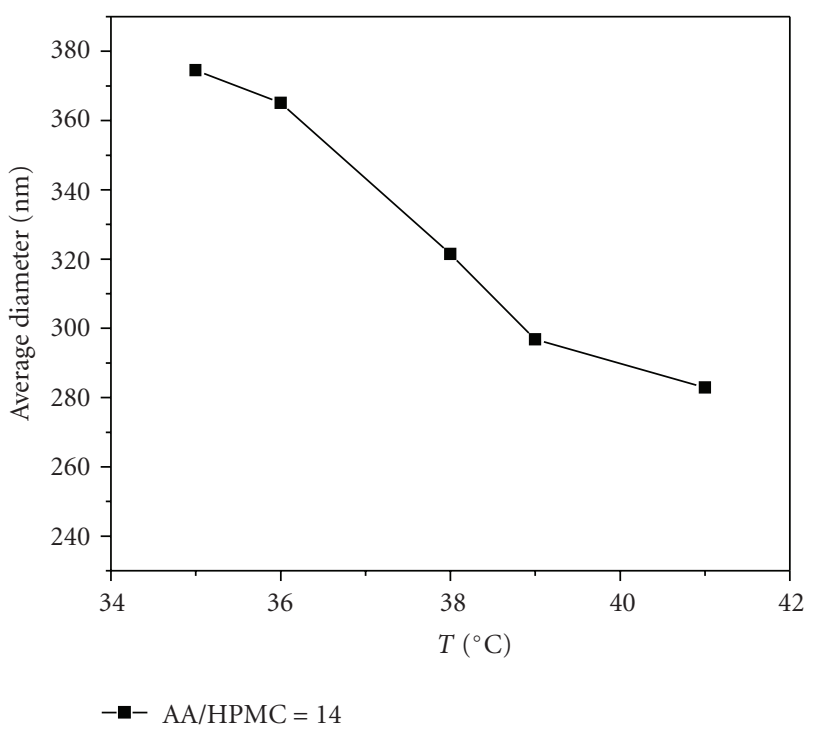

(b)

FIGURE 7: Average diameter of the HPMC/PAA nanoparticles as a function of temperature: the mass ratio of AA to HPMC is (a) 10, (b) 14 (the concentration of HPMC is $0.5 \mathrm{wt} \%$ ).

The size of the HPMC/PAA complex nanoparticles at low and high temperatures has been studied by dynamic light scattering using Malvern Instruments Zetasizer Nano series instrument (ZS90). The sample was allowed to reach equilibrium for $30 \mathrm{~min}$ after adjusting each temperature. The average diameters of the particles decrease as the environmental temperature increases within the range of temperatures studied as seen in Figure 7. It further shows that the HPMC/PAA nanogels undergo a volume phase transition in water from the swollen state to the collapsed state as temperature increases. The nanogels in water swell when the temperature is lower than their volume phase transition temperature. As the temperature was above their volume phase transition temperature (the volume phase transition temperatures seen in Figure 6), water became a relatively poor solvent and escaped from the nanospheres. HPMC polymer chains began to feel the hydrophobic attraction of each other, and the loose surface tangling chains collapsed, resulting in a smaller diameter measured by DLS. This indicates that there is not any particle aggregation at the higher temperatures which could be possibly due to the steric interactions between these particles. Due to the temperature responsive volume change, the suitable transition temperature around $38^{\circ} \mathrm{C}$ under the physiological condition that corresponds to the temperature as a person gets a fever, biocompatibility of the HPMC, and nanosize, the HPMC-PAA nanogels could be particularly useful in controlled drug delivery applications [20].

\section{Conclusion}

The phase transition behavior of hydroxypropyl methylcellulose (HPMC) and acrylic acid (AA) system in water has been investigated. The LCST of the complex shifted to much lower temperature than that of the pure HPMC. The decrease of the phase transition temperature is attributed to strong hydrogen bonding between the HPMC and AA. The LCST of the complexes decreased with the increase of the concentration of acrylic acid monomer. The study of HPMC and AA system leads to a new method for the synthesis of surfactantfree nanogels at the lower temperature, and HPMC-PAA nanogels have been synthesized by AA monomer polymerization. As the reaction temperature just reached LCST, the aggregated chains form stable nanospheres due to intermicelle electrostatic repulsion. The phase transition of the HPMC-PAA nanogels occurs at about $38^{\circ} \mathrm{C}$ as the mass ratio of AA to HPMC is about 14, which corresponds to a fever temperature of the human body. Combining the temperature-responsive volume change, biocompatibility of the HPMC and PAA, and nanosize, the HPMC-PAA nanogels could be particularly useful in controlled drug delivery applications.

\section{References}

[1] R. S. Yao, Q. D. You, P. J. Liu, and Y. F. Xu, "Synthesis and pHinduced phase transition behavior of PAA/PVA nanogels in aqueous media," Journal of Applied Polymer Science, vol. 111, no. 1, pp. 358-362, 2009.

[2] T. Okano, "Molecular design of temperature-responsive polymers as intelligent materials," Advances in Polymer Science, vol. 110, pp. 178-197, 1993.

[3] M. Koussathana, P. Lianos, and G. Staikos, "Investigation of hydrophobic interactions in dilute aqueous solutions of hydrogen-bonding interpolymer complexes by steadystate and time-resolved fluorescence measurements," Macromolecules, vol. 30, no. 25, pp. 7798-7802, 1997.

[4] X. D. Huang and S. H. Goh, "Interpolymer complexes through hydrophobic interactions: $\mathrm{C}_{60}$-end-capped poly(ethylene oxide)/poly(methacrylic acid) complexes," Macromolecules, vol. 33, no. 23, pp. 8894-8897, 2000. 
[5] B. S. Lele and A. S. Hoffman, "Mucoadhesive drug carriers based on complexes of poly(acrylic acid) and PEGylated drugs having hydrolysable PEG-anhydride-drug linkages," Journal of Controlled Release, vol. 69, no. 2, pp. 237-248, 2000.

[6] Y. Dan, S. Chen, Y. Zhang, and F. Xiang, "Viscosity enhancement of complexed solutions formed through the complexation of nonionic water-soluble polymers with chemically complementary structures in aqueous media," Journal of Polymer Science. Part B, vol. 38, no. 8, pp. 1069-1077, 2000.

[7] B. C. Shin, M. S. Jhon, H. B. Lee, and S. H. Yuk, " $\mathrm{pH} /$ temperature dependent phase transition of an interpenetrating polymer network: anomalous swelling behavior above lower critical solution temperature," European Polymer Journal, vol. 34, no. 11, pp. 1675-1681, 1998.

[8] K. Karayanni and G. Staikos, "Study of the lower critical solution temperature behaviour of poly(vinyl methyl ether) aqueous solutions in the presence of poly(acrylic acid). The role of interpolymer hydrogen bonding interaction," European Polymer Journal, vol. 36, no. 12, pp. 2645-2650, 2000.

[9] O. Nikolaeva, T. Budtova, V. Alexeev, and S. Frenkel, "Interpolymer complexation between polyacrylic acid and cellulose ethers: formation and properties," Journal of Polymer Science. Part B, vol. 38, no. 10, pp. 1323-1330, 2000.

[10] O. V. Nikolaeva, T. V. Budtova, L. M. Kalyuzhnaya, N. G. Bel'nikevich, E. N. Vlasova, and S. Y. Frenkel', "Intermolecular interactions in the mixtures of semidilute aqueous solutions of poly(acrylic acid) and cellulose ethers," Polymer Science. Series A, vol. 41, no. 7, pp. 771-776, 1999.

[11] T. V. Budtova, I. E. Suleimenov, and Y. S. Frenkel, "Interpolymer complex formation of some nonionogenic polymers with linear and crosslinked polyacrylic acid," Journal of Polymer Science. Part A, vol. 32, no. 2, pp. 281-284, 1994.

[12] X. Lu, Z. Hu, and J. Schwartz, "Phase transition behavior of hydroxypropylcellulose under interpolymer complexation with poly(acrylic acid)," Macromolecules, vol. 35, no. 24, pp. 9164-9168, 2002.

[13] J. Gao, G. Haidar, X. Lu, and Z. Hu, "Self-association of hydroxypropylcellulose in water," Macromolecules, vol. 34, no. 7, pp. 2242-2247, 2001.

[14] X. Xia, S. Tang, X. Lu, and Z. Hu, "Formation and volume phase transition of hydroxypropyl cellulose microgels in salt solution," Macromolecules, vol. 36, no. 10, pp. 3695-3698, 2003.

[15] X. Lu, Z. Hu, and J. Gao, "Synthesis and light scattering study of hydroxypropyl cellulose microgels," Macromolecules, vol. 33, no. 23, pp. 8698-8702, 2000.

[16] Z. Hu, X. Lu, J. Gao, and C. Wang, "Polymer gel nanoparticle networks," Advanced Materials, vol. 12, no. 16, pp. 1173-1176, 2000.

[17] G. A. Burdock, "Safety assessment of hydroxypropyl methylcellulose as a food ingredient," Food and Chemical Toxicology, vol. 45, no. 12, pp. 2341-2351, 2007.

[18] S. Yang, Y. Zhang, L. Wang et al., "Composite thin film by hydrogen-bonding assembly of polymer brush and poly(vinylpyrrolidone)," Langmuir, vol. 22, no. 1, pp. 338-343, 2006.

[19] Y. He, B. Zhu, and Y. Inoue, "Hydrogen bonds in polymer blends," Progress in Polymer Science, vol. 29, no. 10, pp. 10211051, 2004.

[20] H. Dai, Q. Chen, H. Qin et al., "A temperature-responsive copolymer hydrogel in controlled drug delivery," Macromolecules, vol. 39, no. 19, pp. 6584-6589, 2006. 

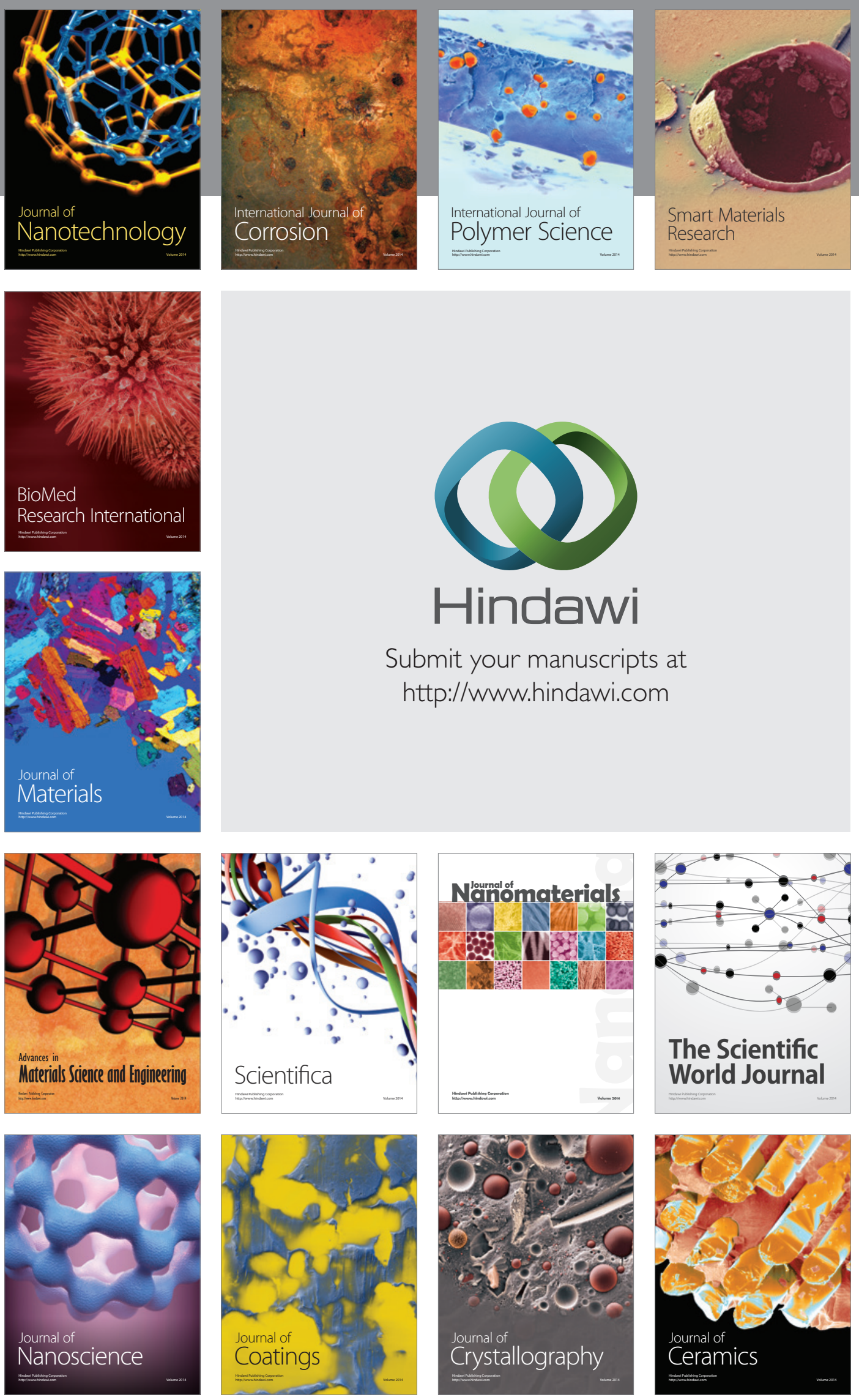

The Scientific World Journal

Submit your manuscripts at

http://www.hindawi.com

\section{World Journal}

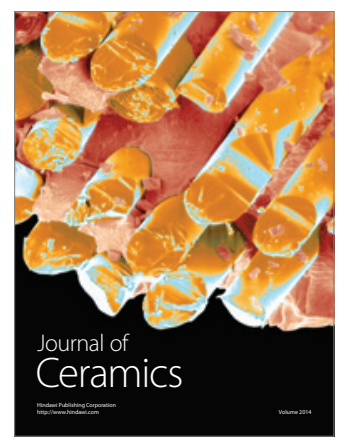

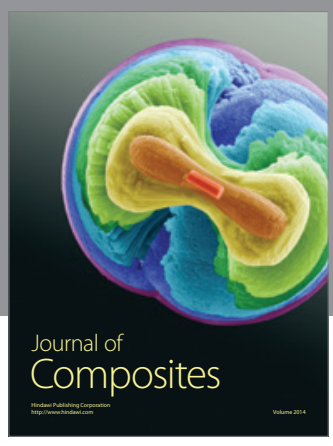
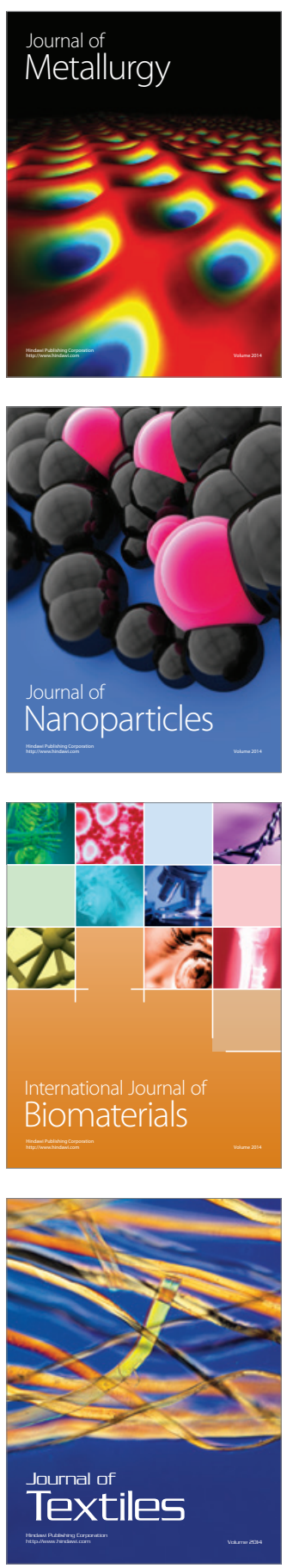